\title{
A Nasopharyngeal Carcinoma Patient With COVID-19 Infection After Immunotherapy: A Case Report and Literature Review
}

\author{
MENGLAN ZHAI and SHENG ZHANG \\ Cancer Center, Union Hospital, Tongji Medical College, Huazhong \\ University of Science and Technology, Wuhan, P.R. China
}

\begin{abstract}
Background/Aim: Novel coronavirus infection in a cancer patient treated with immunotherapy, requires high attention. Case Report: Clinical and radiological data were obtained from the electronic medical record. Pharynx swab was tested for severe acute respiratory syndrome coronavirus 2 (SARS-CoV-2) RNA by reverse transcription-polymerase chain reaction (RT-PCR). The nasopharyngeal carcinoma patient developed fever on the third day after chemotherapy and immunotherapy. Laboratory examination showed lymphocytopenia. On the sixth day, chest computed tomography (CT) images showed bilateral scattered groundglass opacities and reticulation. Pharynx swab was positive for SARS-CoV-2 nucleic acid and the patient was confirmed as having Coronavirus Disease 2019 (COVID-19). Unfortunately, despite aggressive treatment after the diagnosis of COVID-19, the patient died quickly. Conclusion: The patient with nasopharyngeal carcinoma in this case developed severe COVID-19 after receiving immunotherapy. For patients treated with immune checkpoint inhibitors (ICIs) in epidemic areas, the safety of ICIs in cancer patients infected with SARSCoV-2 should be considered.
\end{abstract}

In recent years, ICIs for programmed cell death 1(PD1)/programmed cell death 1 ligand $1(\mathrm{PD}-\mathrm{L} 1)$ and cytotoxic T-cell lymphocyte-4(CTLA-4)pathways have become the most promising tumor immunotherapy strategy (1), which play an important role in regulating self-tolerance and immune homeostasis by blocking the corresponding signal

This article is freely accessible online.

Correspondence to: Sheng Zhang, Cancer Center, Union Hospital, Tongji Medical College, Huazhong University of Science and Technology, Wuhan, 430022, P.R. China. Tel: +86 13971442699, Fax: +8602765659931, e-mail: tonydppx@hotmail.com

Key Words: SARS-CoV-2, COVID-19, immune checkpoint inhibitors, immune checkpoint inhibitor-related pneumonitis, severe pneumonia. pathway. In addition, less well appreciated is the fact that immune checkpoint pathways also regulate antiviral immune responses and are therefore modulated by a number of viruses. Up-regulation of PD-1 and its ligands PD-L1 and PD-L2 is observed during acute virus infection. Experimental evidence suggests that insufficient signaling through the PD-1 pathway promotes immunopathology during acute infection by exaggerating primary $\mathrm{T}$ cell responses (2). Since December 2019, the novel coronavirus infection pandemic has become one of the common threats facing the world. Liang et al. (3) reported that cancer patients might have an increased risk of COVID-19 infection and a poor prognosis. It was also pointed out that cancer patients with COVID-19 who have recently been treated with anti-cancer therapy had a higher risk of severe events. Herein, we report a case of novel coronavirus infection in a nasopharyngeal carcinoma patient treated with anti-PD-1 monoclonal antibody to provide a reference for the safe use of ICIs during epidemic transmission.

\section{Case Presentation}

A 50-year-old man without known significant medical and smoking history, was admitted to hospital with nasopharyngeal carcinoma (cT4N2Mx, suspected liver metastasis). He was treated with gemcitabine-cisplatin (gemcitabine $=1,000 \mathrm{mg} / \mathrm{m}^{2}$ on days 1 and 8 ; cisplatin $=75$ $\mathrm{mg} / \mathrm{m}^{2}$ on days $1-2$ ) plus anti-PD-1 monoclonal antibody Sintilimab (200 $\mathrm{mg}$ on day 1) therapy for the first time. On the third day after treatment, he developed fever, but there were no symptoms such as dry cough and dyspnea. Physical examination showed bilateral cervical lymph node enlargement. Previous medical histories include chronic viral hepatitis $\mathrm{b}$ and diabetes. Considering the prevalence of the novel coronavirus epidemic, laboratory tests showed that the white blood cell count was normal $(7.21 \mathrm{G} / \mathrm{L}, 3.5-9.5 \mathrm{G} / \mathrm{L})$, neutrophils increased $(6.85 \mathrm{G} / \mathrm{L}, 1.8-6.3 \mathrm{G} / \mathrm{L})$, and the absolute value of lymphocytes decreased $(0.35 \mathrm{G} / \mathrm{L}, 1.1-3.2$ $\mathrm{G} / \mathrm{L})$. IL-6 increased to $32.49 \mathrm{pg} / \mathrm{ml}$ and IL-10 increased to $6.77 \mathrm{pg} / \mathrm{ml}$. CD4/CD8 ratio (3.54) was increased. C-reactive 
protein $(70.4 \mathrm{mg} / \mathrm{l})$ was increased, procalcitonin was normal (less than $0.13 \mu \mathrm{g} / \mathrm{l}$ ), and erythrocyte sedimentation rate was increased at $25 \mathrm{~mm} / \mathrm{h}$. Detection of respiratory pathogen spectrum antibody IgM (Mycoplasma pneumoniae IgM, chlamydia pneumoniae $\operatorname{IgM}$, respiratory syncytial virus IgM, adenovirus $\operatorname{IgM}$, coxsackievirus B-IgM) were all negative. Nucleic acids of three respiratory viruses (influenza A virus RNA, influenza B virus RNA, respiratory syncytial virus RNA) were all negative by nasal swabs. On the sixth day after treatment, the patient still had fever and new symptoms of dry cough and dyspnea. Chest CT images showed bilateral scattered ground-glass opacities and reticulation mainly distributed in a sub-pleural area, which were highly suspected to be caused by viral infection, as shown in Figure 1. The pharynx swab was positive for SARS-CoV-2 nucleic acid by RT-PCR assay approved by the China Food and Drug Administration. He was then referred to a designated critical hospital (Jin Yin-tan Hospital, Wuhan, PR China) for treatment as a confirmed case of COVID-19. After he was transferred to the designated hospital, the fever and dry cough persisted, and dyspnea worsened.

He was followed up by telephone, as his laboratory examination results and lung CT images in the designated hospital were not available. He received aggressive antiviral (ribavirin, arbidol, and hydroxychloroquine), glucocorticosteroids (methylprednisolone $2 \mathrm{mg} / \mathrm{kg}$ per day for 7 days), respiratory support and other supportive treatment. Antifungal (fluconazole) and wide-spectrum antibiotics (moxifloxacin meropenem) were used to prevent secondary infections. Unfortunately, he died 16 days after treatment. Written informed consent was obtained from the patient's next to kin for publication of this case report.

\section{Discussion}

During the COVID-19 pandemic, the management of cancer patients treated with ICIs presents a major challenge. ICIs may enhance $\mathrm{T}$ cell activity, but it will damage the normal lung tissue at the same time, leading to ICI-related pneumonitis. Available literature reports that there might be potential overlap between ICI-related pneumonitis and COVID-19 associated interstitial pneumonia (4). The incidence of ICI-related pneumonitis in patients receiving anti-PD-1/PD-L1 monotherapy and anti-PD-1 combined with anti-CTLA-4 therapy were $2.5-5 \%$ and $7-10 \%$, respectively (5). The median time to onset of ICI-related pneumonitis was 2.8 months (6). The febrile symptoms of this patient appeared within 1 week after the first treatment with the PD1 inhibitor, and ICI-related pneumonitis was basically excluded. His fever might also have been caused by neutropenia after chemotherapy. In severe cases, it would even be complicated with bacterial pneumonia. However, his neutrophil count was normal. Combined with the positive result of the novel coronavirus nucleic acid test, SARS-CoV2 infection was confirmed.

Studies have indicated that immunotherapy increases the risk of severe COVID-19 events $(7,8)$. Bonomi et al. (9) and Di Noia et al. (10) separately reported that a non-small cell lung cancer patient with long-term anti-PD-1 monotherapy died quickly after infected with COVID-19. Seven of the 28 cancer patients infected with COVID-19 reported by Zhang et al. (11) were lung cancer patients. Among them, one patient received anti-CTLA-4/anti-PD1 combination therapy and chemotherapy combined with anti-PD-1 monotherapy therapy 3 months and 14 days before onset of symptoms, respectively. She developed a severe case of COVID-19. Wu et al. (12) reported that 11 cancer patients with COVID-19 received immunotherapy, of whom 7 developed severe disease and 4 died of COVID-19. Meanwhile, it was pointed out that patients who received more than 3 cycles of immunotherapy had a higher risk of severe events, but the difference was not statistically significant, probably because of the very small sample. Currently, there is no specific drug for COVID-19, and the treatment is mainly antiviral, antibacterial, oxygen therapy and other comprehensive treatment (13). In this study, the patient received immunotherapy before getting SARS-CoV-2. He finally died of ineffectiveness despite active treatment. Several studies have shown that the severity of COVID-19 is independent of immunotherapy (14-16). Besides, findings have indicated that there is no correlation between the interval of the last use of ICIs and the severity of COVID-19 (12, 15). Szabados et al. (17) reported four cancer patients who had received immunotherapy before infected with COVID-19 (two patients with metastatic urothelial cancer and another two with metastatic renal cancer). No severe events occurred whether they were previously treated with anti-PD-1 monotherapy or anti-PD-1 combined with anti-CTLA-4. Li et al. (18) reported that a lymphoma patient undergoing intensive immunochemotherapy developed grade 4 myelosuppression with SARS-CoV-2 infection. After comprehensive treatment, his COVID-19 was cured.

The severity of COVID-19 in cancer patients with immunotherapy may be related to the mechanism of lung injury caused by immunotherapy and SARS-COV-2 infection. However, to date, the mechanism of lung injury caused by SARS-CoV-2 infection is not yet entirely clear. In this case, the absolute value of lymphocytes decreased and most inflammatory factors increased. This result suggested that COVID-19 might be related to the "cytokine storm" caused by the overreaction of the immune system caused by viral infection and the release of a large amount of cytokines, tumor necrosis factors and others (19). Glucocorticoids can suppress inflammatory cytokine storms and can be used for ICI-related pneumonitis and COVID-19 (20). Survival of this patient failed to improve after low-dose 

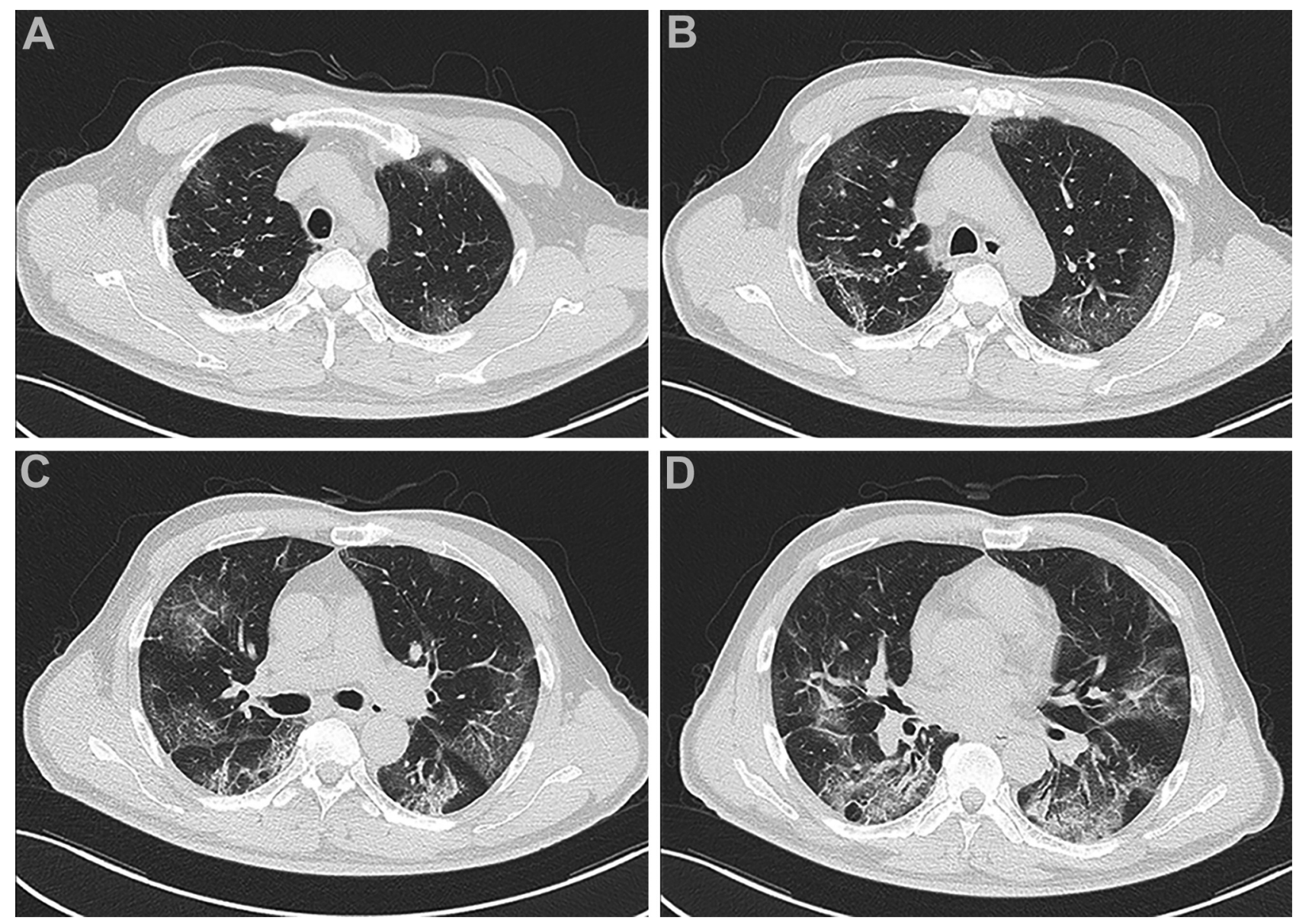

Figure 1. Chest computed tomography (CT) showed bilateral scattered ground-glass opacities and reticulation mainly distributed in a sub-pleural area.

glucocorticoid therapy, which might be related to the function of glucocorticoid in inhibiting the body's immune response and delaying virus clearance (21). ICI exposure may enhance the cytokine storm associated with COVID-19 and further aggravate COVID-19 (22). PD-1 inhibitors can enhance the antiviral immune response in the process of virus infection by inhibiting PD-1 signaling pathway (23), which can theoretically improve the disease. Therefore, based on the current researches, it cannot be proved that severe pneumonia caused by SARS-COV-2 infection is related to immunotherapy.

\section{Conclusion}

The patient with nasopharyngeal carcinoma in this case developed severe COVID-19 after receiving immunotherapy. However, the effect of immunotherapy on COVID-19 remains unclear. Therefore, under the circumstances that the spread of the epidemic has not been completely controlled, further studies are needed to explore the safety of ICIs in cancer patients infected with COVID-19.

\section{Conflicts of Interest}

The Authors declare that the research was conducted in the absence of any commercial or financial relationships that could be construed as a potential conflict of interest.

\section{Authors' Contributions}

SZ made substantial contributions to the conception and design of the work. SZ and MZ made substantial contributions to the laboratory analysis and interpretation of data. SZ and MZ made substantial contributions to the acquisition of clinical data and their interpretation. SZ and MZ were involved in drafting the manuscript. $\mathrm{SZ}$ was involved in critically revising the manuscript for important intellectual content. SZ and MZ read and approved the final manuscript.

\section{Acknowledgements}

The Authors would like to thank the health care workers who are involved in the fight against COVID-19. 


\section{References}

1 Ghahremanloo A, Soltani A, Modaresi SMS and Hashemy SI: Recent advances in the clinical development of immune checkpoint blockade therapy. Cell Oncol (Dordr) 42(5): 609-626, 2019. PMID: 31201647. DOI: 10.1007/s13402-019-00456-w

2 Schonrich G and Raftery MJ: The PD-1/PD-L1 axis and virus infections: A delicate balance. Front Cell Infect Microbiol 9: 207, 2019. PMID: 31263684. DOI: 10.3389/fcimb.2019.00207

3 Liang W, Guan W, Chen R, Wang W, Li J, Xu K, Li C, Ai Q, Lu W, Liang H, Li S and He J: Cancer patients in SARS-CoV-2 infection: a nationwide analysis in China. Lancet Oncol 21(3): 335-337, 2020. PMID: 32066541. DOI: 10.1016/S14702045(20)30096-6

4 Bersanelli M: Controversies about COVID-19 and anticancer treatment with immune checkpoint inhibitors. Immunotherapy 12(5): 269-273, 2020. PMID: 32212881. DOI: 10.2217/imt2020-0067

5 Naidoo J, Wang X, Woo KM, Iyriboz T, Halpenny D, Cunningham J, Chaft JE, Segal NH, Callahan MK, Lesokhin AM, Rosenberg J, Voss MH, Rudin CM, Rizvi H, Hou X, Rodriguez K, Albano M, Gordon RA, Leduc C, Rekhtman N, Harris B, Menzies AM, Guminski AD, Carlino MS, Kong BY, Wolchok JD, Postow MA, Long GV and Hellmann MD: Pneumonitis in patients treated with anti-programmed death1/programmed death ligand 1 therapy. J Clin Oncol 35(7): 709717, 2017. PMID: 27646942. DOI: 10.1200/JCO.2016.68.2005

6 Dirks J, Egli A, Sester U, Sester M and Hirsch HH: Blockade of programmed death receptor-1 signaling restores expression of mostly proinflammatory cytokines in anergic cytomegalovirusspecific T cells. Transpl Infect Dis 15(1): 79-89, 2013. PMID: 23176118. DOI: $10.1111 /$ tid.12025

7 Robilotti EV, Babady NE, Mead PA, Rolling T, Perez-Johnston R, Bernardes M, Bogler Y, Caldararo M, Figueroa CJ, Glickman MS, Joanow A, Kaltsas A, Lee YJ, Lucca A, Mariano A, Morjaria S, Nawar T, Papanicolaou GA, Predmore J, Redelman-Sidi G, Schmidt E, Seo SK, Sepkowitz K, Shah MK, Wolchok JD, Hohl TM, Taur Y and Kamboj M: Determinants of COVID-19 disease severity in patients with cancer. Nat Med 26(8): 1218-1223, 2020. PMID: 32581323. DOI: 10.1038/s41591-020-0979-0

8 Lara OD, O'Cearbhaill RE, Smith MJ, Sutter ME, Knisely A, McEachron J, Gabor LR, Jee J, Fehniger JE, Lee YC, Isani SS, Wright JD and Pothuri B: COVID-19 outcomes of patients with gynecologic cancer in New York City. Cancer, 2020. PMID: 32729142. DOI: $10.1002 /$ cncr.33084

9 Bonomi L, Ghilardi L, Arnoldi E, Tondini CA and Bettini AC: A rapid fatal evolution of coronavirus disease-19 in a patient with advanced lung cancer with a long-time response to nivolumab. J Thorac Oncol 15(6): e83-e85, 2020. PMID: 32243919. DOI: $10.1016 /$ j.jtho.2020.03.021

10 Di Noia V, D'Aveni A, Squadroni M, Beretta GD and Ceresoli GL: Immune checkpoint inhibitors in SARS-CoV-2 infected cancer patients: the spark that ignites the fire? Lung Cancer 145: 208-210, 2020. PMID: 32439211. DOI: 10.1016/j.lungcan.2020.05.006

11 Zhang L, Zhu F, Xie L, Wang C, Wang J, Chen R, Jia P, Guan HQ, Peng L, Chen Y, Peng P, Zhang P, Chu Q, Shen Q, Wang Y, Xu SY, Zhao JP and Zhou M: Clinical characteristics of COVID19-infected cancer patients: a retrospective case study in three hospitals within Wuhan, China. Ann Oncol 31(7): 894-901, 2020. PMID: 32224151. DOI: 10.1016/j.annonc.2020.03.296
12 Wu Q, Chu Q, Zhang H, Yang B, He X, Zhong Y, Yuan X, Chua MLK and Xie C: Clinical outcomes of coronavirus disease 2019 (COVID-19) in cancer patients with prior exposure to immune checkpoint inhibitors. Cancer Commun (Lond) 40(8): 374-379, 2020. PMID: 32666636. DOI: 10.1002/cac2.12077

13 National Health Commission of the People's Republic of China: Diagnosis and treatment of pneumonia caused by novel coronavirus infection (trial version 7). Available at: http://www.nhc.gov.cn/xcs/zhengcwj/202003/46c9294a7dfe4cef8 Odc7f5912eb1989.shtml [Last accessed on March 4, 2020]

14 Lee LYW, Cazier JB, Starkey T, Turnbull CD; UK Coronavirus Cancer Monitoring Project Team, Kerr R and Middleton G: COVID-19 mortality in patients with cancer on chemotherapy or other anticancer treatments: a prospective cohort study. Lancet 395(10241): 1919-1926, 2020. PMID: 32473682. DOI: 10.1016/ S0140-6736(20)31173-9

15 Luo J, Rizvi H, Egger JV, Preeshagul IR, Wolchok JD and Hellmann MD: Impact of PD-1 blockade on severity of COVID19 in patients with lung cancers. Cancer Discov 10(8): 1121-1128, 2020. PMID: 32398243. DOI: 10.1158/2159-8290.CD-20-0596

16 van Dam PA, Huizing M, Mestach G, Dierckxsens S, Tjalma W, Trinh XB, Papadimitriou K, Altintas S, Vermorken J, Vulsteke C, Janssens A, Berneman Z, Prenen H, Meuris L, Vanden Berghe W, Smits E and Peeters M: SARS-CoV-2 and cancer: Are they really partners in crime? Cancer Treat Rev 89: 102068, 2020. PMID: 32731090. DOI: 10.1016/j.ctrv.2020.102068

17 Szabados B, Abu-Ghanem Y, Grant M, Choy J, Bex A and Powles T: Clinical characteristics and outcome for four SARSCoV-2-infected cancer patients treated with immune checkpoint inhibitors. Eur Urol 78(2): 276-280, 2020. PMID: 32534910. DOI: $10.1016 /$ j.eururo.2020.05.024

18 Li Q, Zhu F, Xiao Y, Liu T, Liu X, Wu G and Zhang L: A primary mediastinal Large B-Cell lymphoma patient with COVID-19 infection after intensive immunochemotherapy: A case report. Front Oncol 10: 924, 2020. PMID: 32574278. DOI: 10.3389 /fonc. 2020.00924

19 Smetana K and Brábek J: Review: Role of interleukin-6 in lung complications in patients with COVID-19: Therapeutic implications. In Vivo 34(3 suppl): 1589-1592, 2020. PMID: 32503815. DOI: 10.21873 /invivo.11947

20 Cain DW and Cidlowski JA: Immune regulation by glucocorticoids. Nat Rev Immunol 17(4): 233-247, 2017. PMID: 28192415. DOI: $10.1038 /$ nri.2017.1

21 Hui DS: Systemic corticosteroid therapy may delay viral clearance in patients with middle east respiratory syndrome coronavirus infection. Am J Respir Crit Care Med 197(6): 700-701, 2018. PMID: 29227752. DOI: 10.1164/rccm.201712-2371ED

22 Mehta P, McAuley DF, Brown M, Sanchez E, Tattersall RS, Manson JJ and HLH Across Speciality Collaboration, UK: COVID-19: consider cytokine storm syndromes and immunosuppression. Lancet 395(10229): 1033-1034, 2020. PMID: 32192578. DOI: 10.1016/S0140-6736(20)30628-0

23 McNally B, Ye F, Willette M and Flano E: Local blockade of epithelial PDL-1 in the airways enhances T cell function and viral clearance during influenza virus infection. J Virol 87: 1291612924, 2013. PMID: 24067957. DOI: 10.1128/JVI.02423-13

Received August 12, 2020

Revised August 27, 2020 Accepted August 28, 2020 